\title{
Neuropsychobiology 1983;9:19
}

\section{Editorial}

The large variety of research in the field of pharmacopsychiatry has, within the last 10-15 years, brought about an enormous spreading out of the spectrum of research, which ranges from psychopharmacology to biological psychiatry including new specialised sections as pharmacopsychology or pharmacoencephalography, as well as infiltrating into other specialities as, e.g., neuro-physiology. The newly concepted Neuropsychobiology tries to counteract the increasing loss of contact of these single special groups by presenting an organ with different areas for four specialities which are strongly interrelated. As a modern and adaptable organ, the journal will not exclude further subgroups from being incorporated into it. 
The section 'Pharmacopsychiatry' remains as hitherto open for publication from all the domains of pharmacopsychiatry, from basic psychopharmacological research to clinical pharmacopsychiatry. The range shall remain as wide as possible, as only a survey of clinically relevant results of basis research, clinical pharmacopsychiatry, and the use of psychopharmaca in other areas as, e.g., in psychotherapy, can offer a comprehensive information for the reader. Differing from the praxis until now of International Pharmacopsychiatry, in future only papers in English will be accepted.

J. Fleischhauer, St. Urban 